\title{
Small Airways Disease, Emphysema, Lung Cancer, Multi-Morbidities and Therapeutic Trials...
}

R. Rodriguez-Roisin, MD, PhD, FRCP, FERS

The last issue of BRN Reviews in 2017 addresses six relevant topics of great interest to respiratory medicine. The first one features 'small airways disease', an inspiring concept closely linked to the early development of chronic obstructive pulmonary disease (COPD) and other chronic obstructive airway diseases, presented by James Hogg and Tillie-Louise Hackett. For those of us who started chest medicine in the early 1970s, it was very soon learnt that 'small airways disease' represented one of the major breakthroughs and challenges of the future understanding of many chronic obstructive airway disorders. To celebrate the half century of its seminal publications, $B R N$ Reviews is most fortunate and honoured to have Prof Hogg as one of the Canadian authors in this opening review of the current issue. Along with other big names, such as Peter Macklem, Jere Mead and William Thurlbeck, Prof Hogg is one of the giants of respiratory medicine involved in the early investigation of 'small airways disease'. Most importantly, he is still an active cutting-edge researcher in chronic obstructive airway disorders. The review further underpins the hypothesis that small conducting airways represent a 'quiet' zone within the lung where disease can accumulate over many years without being noticed, underlining the mechanisms why these small conducting airways are susceptible to the development of COPD.

The next article focuses on the complex interplay between pulmonary emphysema and COPD. The two Spanish authors, Juan Pablo de Torres and Ciro Casanova, are very productive international experts in the field, which is beautifully covered through the review of the prevalence of emphysema in, and its impact on, COPD and its association with other important patient-related outcomes, such as exacerbations, lung function decline, comorbidities and mortality. The authors highlight that emphysema is associated with rapid $\mathrm{FEV}_{1}$ decline, increased frequency of exacerbations and allcause mortality in COPD, an association independent of the severity of airflow limitation.

The third contribution, by an Italian team integrated by Bianca Beghé, Enrico Clini and Leonardo M. Fabbri, focuses on the presence

DOI: 10.23866/BRNRev:2017-0057 
of chronic respiratory abnormalities in the multi-morbid frail elderly individuals that affect more than two thirds of the population, causing both physical and mental health impairment that almost invariably jeopardize health-related quality of life in these subjects. It is of note that the clinical approach of an individual with any of the chronic diseases that are part of the concept of multi-morbidity does not just represent the management of that single presenting index disease, but must include the active search and proper management and therapy of other concomitant chronic diseases. Its senior author, Prof Fabbri, was one of the first international renowned scientists to draw the attention to the international community 10 years ago on this field, dubbed 'chronic systemic inflammatory disease' ${ }^{1}$.

The next article concentrates on an extensive state-of-the art review on lung cancer management, addressed by three international scholars with solid experience in the field, i.e. Giorgio Scagliotti, Paulo Bironzo and Antoni Rosell. Among the key aspects pointed out, lung cancer control to identify and characterize the increasing number of risk factors, such as air pollution induced by the inhalation of aggressive fine particles, the importance of some screening programs, such as computed tomography nodule volumetry and new bronchoscopy approaches, and new mortality scores are three of the most relevant. Likewise, the review rightly addresses the new randomized controlled trials (RCT) that involve innovative therapeutic attempts using novel biologics.

The last two articles are focused on therapeutic aspects. The first one reviews some of the most relevant pharmacological RCTs in COPD by two British scholars, Peter Calverley and Paul Walker, the former one of the most brilliant and productive researchers in COPD over the last 40 years. Fueled by inspiring subheadings, such as 'Big is beautiful but simple is best', 'You don't always get what you want ...', '... But you might just get what you need', and others, this authorized review critically distils some of the most enthralling original RCTs designed in patients with moderate-to-very severe COPD over the last decade, most of them having Prof Calverley as first author.

Lastly, the second therapeutic contribution reviews one of the most relevant current nonpharmacological tools to manage and treat episodes of acute hypercapnic respiratory failure (AHRF), more specifically non invasive ventilation (NIV). Its British author, Mark Elliott, has made important contributions in the field since the early 2000s of its implementation to handle all sorts of acute respiratory conditions. As he rightly pointed out, NIV is currently part of the mainstream of all sorts of acute respiratory insufficiency and critical care medicine, including a well-developed and evidence-based role in the management of exacerbations of COPD. It is also strongly and successfully recommended in other conditions provoking AHRF, and the first line intervention in the vast majority of cases.

I really hope that you will enjoy this last issue of BRN Reviews in 2017. Indeed, it certainly features very germane and timely concepts of respiratory medicine signed by a group of current talented scientists.

\section{REFERENCES}

1. Fabbri L. Leonardo Fabbri: investigating the complexity of COPD. Interview by Kelly Morris. Lancet 2009; 374: 679 . 